\title{
Two-year outcomes in initial survivors with acute liver failure: results from a prospective, multicentre study
}

Robert J. Fontana ${ }^{1}$, Caitlyn Ellerbe ${ }^{2}$, Valerie E. Durkalski ${ }^{2}$, Amol Rangnekar ${ }^{1}$, Rajender K. Reddy ${ }^{3}$, Todd Stravitz ${ }^{4}$, Brendan McGuire, ${ }^{5}$ Timothy Davern ${ }^{6}$, Adrian Reuben ${ }^{7}$, Iris Liou ${ }^{8}$, Oren Fix ${ }^{9}$, Daniel R. Ganger ${ }^{10}$, Raymond T. Chung ${ }^{11}$, Mike Schilsky ${ }^{12}$, Steven Han ${ }^{13}$, Linda S. Hynan ${ }^{14}$, Corron Sanders ${ }^{14}$ and William M. Lee ${ }^{14}$ for the US Acute Liver Failure Study Group

1 Division of Gastroenterology, Department of Internal Medicine, University of Michigan Medical Center, Ann Arbor, MI, 48109-0362, USA

2 Division of Biostatistics, Department of Public Health Sciences, Medical University of South Carolina, Charleston, SC, USA

3 Department of Medicine, University of Pennsylvania, Philadelphia, PA, USA

4 Department of Medicine, Virginia Commonwealth University, Richmond, VA, USA

5 Department of Medicine, University of Alabama at Birmingham, Birmingham, AL, USA

6 California Pacific Medical Center, San Francisco, CA, USA

7 Department of Internal Medicine, Medical University of South Carolina, Charleston, SC, USA

8 Department of Internal Medicine, University of Washington, Seattle, WA, USA

9 Department of Internal Medicine, University of California San Francisco, San Francisco, CA, USA

10 Division of Gastroenterology, Northwestern University, Chicago, IL, USA

11 Massachusetts General Hospital, Boston, MA, USA

12 Section of Transplantation and Immunology, Yale University, New Haven, CT, USA

13 Department of Internal Medicine, University of California, Los Angeles, CA, USA

14 Division of Digestive \& Liver Diseases, Department of Internal Medicine, University of Texas Southwestern, Dallas, TX, USA

\section{Keywords}

age - cerebral oedema - liver transplantation

- prognosis - regeneration

\section{Abbreviations \\ ALF, acute liver failure; ALFSG, acute liver failure study group; ALT, alanine aminotransferase; APAP, acetaminophen; $B$ virus; ICP, intracranial pressure; IDU, intravenous drug use; INR, international model for end-stage liver disease; SS, spontaneous survivors. \\ Correspondence \\ Robert J. Fontana, MD Professor of Medicine 3912 Taubman Center Ann Arbor, Ml 48109-0362, USA \\ Tel: +17349364780 \\ Fax: +1 7349367392 \\ e-mail: rfontana@med.umich.edu} DILI, drug-induced liver injury; HBV, hepatitis normalized ratio; LT, liver transplant; MELD,

Received 12 March 2014

Accepted 1 July 2014

DOI:10.1111/liv.12632

Liver Int. 2015; 35: 370-380

\begin{abstract}
Background \& Aims: The long-term clinical outcomes in initial survivors with acute liver failure (ALF) are not well known. The aim of this study was to provide an overview of the 2-year clinical outcomes among initial survivors and liver transplant (LT) recipients that were alive 3 weeks after enrolment in the Acute Liver Failure Study Group (ALFSG). Methods: Outcomes in adult ALFSG patients that were enrolled between 1998 and 2010 were reviewed. Results: Two-year patient survival was significantly higher in the 262 LT recipients $(92.4 \%)$ compared to the 306 acetaminophen (APAP) spontaneous survivors (SS) $(89.5 \%)$ and 200 non-APAP SS (75.5\%) $(P<0.0001)$. The causes of death were similar in the three groups but the time to death was significantly longer in the LT recipients $(P<0.0001)$. Independent predictors of 2-year mortality in the APAP group were a high serum phosphate level and patient age $(\mathrm{c}$-statistic $=0.65(0.54,0.76))$, patient age and days from jaundice to ALF onset in the non-APAP group (cstatistic $=0.69(0.60,0.78))$, and patient age, days from jaundice, and higher coma grade in the LT recipients (c-statistic $=0.74(0.61,0.87))$. The LT recipients were significantly more likely to be employed and have a higher educational level $(P<0.05)$. Conclusions: Two-year outcomes in initial survivors of ALF are generally good but non-APAP patients have a significantly lower survival which may relate to pre-existing medical comorbidities. Spontaneous survivors with APAP overdose experience substantial morbidity during follow-up from ongoing psychiatric and substance abuse issues.
\end{abstract}

Acute liver failure (ALF) is a rare but dramatic illness associated with a high rate of short-term morbidity and mortality $(1,2)$. The likelihood of an early adverse out- come including the need for emergency liver transplantation (LT) is, in part, related to the aetiology of ALF, peak grade of encephalopathy and development of 
multi-organ failure (1-4). Results of several studies have demonstrated a lower 1-year patient survival in ALF LT recipients compared to those with cirrhosis undergoing LT because of the emergency nature of the surgery, cerebral oedema, and frequent need for life support in the $\operatorname{ALF}$ patients $(2,3)$. In addition, a higher rate of allograft dysfunction including acute and chronic rejection, de novo autoimmune hepatitis, and recurrent graft failure have been demonstrated during follow-up in ALF LT recipients (5-7). Furthermore, variable degrees of neurological sequelae in long-term survivors of ALF have been reported but the number of patients evaluated has been small $(8,9)$.

The USA Acute Liver Failure Study Group (ALFSG) is a consortium of 13 academic medical centres that is conducting an ongoing observational registry study of adult ALF patients (2). Important insights into the aetiology, disease-specific prognosis and clinical correlates of early adverse outcomes have been reported $(1,2)$. In addition to collecting detailed clinical, demographical, and outcome data up to 3 weeks after enrolment, this study includes a prospective assessment of patient outcomes at 1 and 2 years after enrolment. This effort has proven useful in better defining the long-term clinical outcomes in specific subgroups of ALF patients $(10,11)$. The aim of this study was to provide an overview on the 2-year clinical outcomes among initial survivors and LT recipients who were alive 3 weeks after enrolment in the ALFSG. Baseline demographics and clinical factors associated with impaired long-term survival were identified as well as the frequency of various clinical complications among the following three groups: acetaminophen (APAP) initial spontaneous survivors (SS), non-APAP initial SS, and LT recipients. We hypothesized that LT recipients would have poorer long-term outcomes because of their more severe illness necessitating emergency surgery. Multivariate models to identify patients at risk of dying within 2 years of follow-up were constructed to provide prognostic data for clinicians.

\section{Patients and methods}

Patient selection

The ALFSG database consists of 1850 adult patients with ALF admitted to one of 13 actively or 10 previously enrolling clinical sites from 1 January 1998 to 1 July 2010. Eligible ALF patients had symptoms of jaundice or illness of $<26$ weeks prior to admission and mental status changes with coagulopathy defined as an international normalized ratio (INR) $>1.5$ without known underlying chronic liver disease. The protocol was reviewed and approved by the Institutional Review Board at all of the participating sites and written informed consent was obtained from the patient's next of kin. In addition to baseline demographics and presenting features, detailed information during the first 7 days of enrolment including imaging, lab, and clinical data, as well as short-term outcome data were obtained through 21 days after admission.

\section{Long-term follow-up protocol and data collection}

In calendar year 2000, the ALFSG observational study was amended to include a 12 -month study visit to be performed at 6-18 months after enrolment as well as a 24-month study visit completed any time after 18 months from enrolment. Data obtained at these visits included incremental medical history, medications, liver biopsy and imaging data, and laboratory variables. In addition, various complications were prospectively tracked including neurological complications. Data were collected at a face to face study visit or via telephone interview and/or chart review. For patients that were lost to follow-up, sites queried the Social Security death index database and reviewed medical records. To be included in the analysis population, patients must have survived to short-term follow-up at 21 days (1138 patients) and either have a recorded death or at least one long-term follow-up form completed 21 days after enrolment (773 patients). For the current analysis, patients were stratified on whether they received a liver transplant (LT), and the 3-week initial spontaneous survivors were further stratified by presence of APAPrelated or other non-APAP aetiology.

\section{Statistical analysis}

SAS software version 9.2 (SAS Institute Inc. Cary, NC, USA) was used to perform statistical analysis. Baseline variables were described using counts and percentages for categorical data, or mean and standard deviations (medians and interquartile ranges) for continuous normal (skewed) data. For variables identified as clinically relevant, statistical tests were performed using chisquare, ANOVA, or Kruskal-Wallis tests. Owing to the low event rate in each of the populations, 2-year mortality was reported using cumulative per cent and tested using a test of independent proportions. Predictors of death were modelled with univariate and multivariate logistic regression, with predictors identified using stepwise elimination based on significance and overall goodness of fit. Variables with a $P<0.20$ on univariate analyses were considered for the multivariable analysis. Results of the modelling are expressed as odds ratios with $95 \%$ confidence intervals. The c-statistic and corresponding $95 \%$ confidence interval are based on an approximation (12).

\section{Results}

There were 1138 adult ALF patients alive at 3 weeks after initial enrolment into the ALFSG registry between 


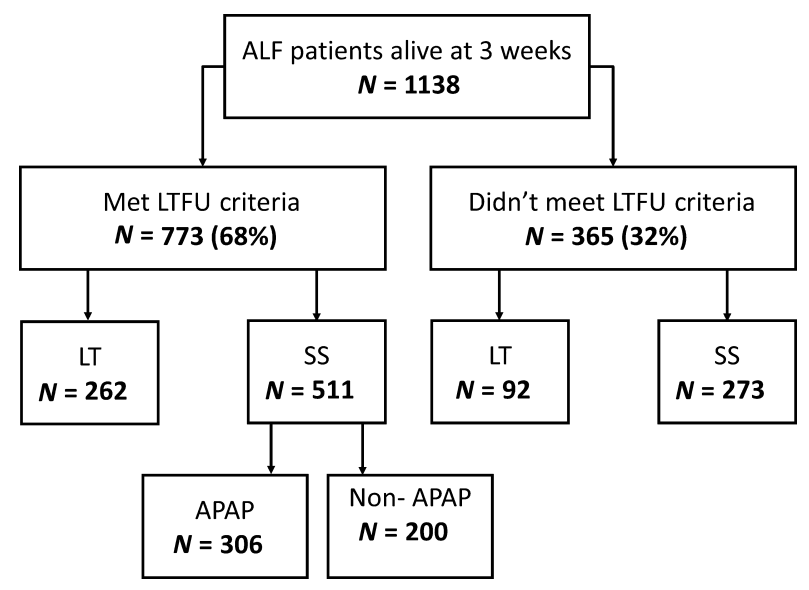

Fig. 1. ALF patients included in the current analysis. Among the $773 \mathrm{ALF}$ patients eligible for this study, there were 262 liver transplant (LT) recipients and 511 initial spontaneous survivors (SS) who were alive 3 weeks after enrolment into the ALFSG registry.

January 1998 and July 2010 (Fig. 1). Long-term followup data were available in $773(68 \%)$ of these patients. Among the 773 patients included in this analysis, there were 262 LT recipients and 511 initial spontaneous survivors (SS) which included 306 with APAP overdose and 200 with ALF because of other non-APAP aetiologies (Table 1). The patients not included in this analysis were similar in baseline demographical and clinical features except that the excluded population was more likely to have experienced APAP overdose (Table S1).

The 306 patients in the APAP initial SS group were younger and significantly more likely to be female, Caucasian, and of non-Hispanic ethnicity compared to the other two patient groups. As expected, the APAP initial survivors had the shortest duration of symptoms, the highest serum aminotransferase levels and lowest bilirubin levels at presentation. The APAP initial survivors were also significantly more likely to have active psychiatric and substance abuse comorbidities at enrolment and a positive urine toxicology screen. A careful review of the cases demonstrated that 116 of these were intentional APAP overdose patients and 159 were non-intentional therapeutic misadventures, while 31 were of unknown intent. The majority of LT recipients were also women and they had a significantly longer duration of symptoms to jaundice, higher total bilirubin and lower serum aminotransferase levels at presentation compared to the other two groups. The frequency of observed aetiologies of ALF in the LT recipients differed when compared to non-APAP initial SS $(P \leq 0.001)$. The LT recipients also had the highest INR, lactate, and model for end-stage liver disease (MELD) scores at presentation. Although the level of hepatic encephalopathy was similar in all three patient groups at admission, the LT recipients were more likely to have an intracranial pressure (ICP) monitor placed, require mechanical intubation, and receive pressors and mannitol during their illness compared to the other two patient groups.

\section{Two-year patient survival}

The 2-year actuarial patient survival was highest in the LT recipients $(92.4 \%)$ followed by the APAP initial SS $(89.5 \%)$ and non-APAP initial SS (75.5\%). Cumulative death rates are presented in Fig. 2. The median time to death in the LT recipients of 223 days (range: 23568 days) was significantly longer compared to the other two patient groups $(P<0.001)$. Overall, the leading causes of death were liver related (17\%), infection/ sepsis $(12 \%)$, and multisystem organ failure (18\%), but $45 \%$ of the deaths were of unknown cause (Table 2). The identified causes of death were similar in the three patients groups.

\section{Predictors of impaired long-term survival}

Multivariate models were constructed to identify baseline variables associated with impaired 2-year survival in each of the three patient groups (Table 3). Univariate analyses of the admission clinical data were used to identify potential variables for model building.

\section{Acetaminophen initial spontaneous survivors}

Older patient age, serum alanine aminotransferase (ALT) as well as elevated serum phosphate, lower arterial $\mathrm{pH}$, and higher initial coma grade were significant predictors of death on univariate analysis. In the multivariate model, age and phosphate remained as consistent predictors of death with an elevated phosphate level indicating higher risk of death (OR: 1.26, 95\% CI: 1.05 , 1.51). The median age of the APAP patients that died during follow-up was 42, while the median age of the long-term survivors was 35 . The median admission phosphate of those that died was $2.75 \mathrm{mg} / \mathrm{dl}$ compared to $2.20 \mathrm{mg} / \mathrm{dl}$ in those that survived. Of note, the proportion of patients with intentional APAP overdose was similar in the long-term survivors and non-survivors $(41 \%$ vs. $42 \%, P=0.93)$ as was the presence of psychiatric comorbidities ( $44 \%$ vs. $56 \%, P=0.19)$.

\section{Non-acetaminophen spontaneous survivor group}

Older patient age, non-Hispanic ethnicity, $<12$ years of education, increased days of jaundice to ALF, serum ALT, higher INR and pressor use were examined as potential predictors of death for the multivariate analysis. Of note, initial coma grade, need for dialysis and intubation were not associated with long-term survival in univariate analyses. In the multivariate model, age and days from jaundice to ALF were the only independent predictors of death in this patient group. The median age of the non-APAP patients who died was 50 vs. 42 in those that survived. The aetiologies of ALF in 
Table 1. Baseline characteristics of the long-term follow-up ALF patient groups

\begin{tabular}{|c|c|c|c|c|c|}
\hline & $N$ & $\begin{array}{l}\text { APAP } \\
\text { initial } \\
\text { survivors } \\
(N=306)\end{array}$ & $\begin{array}{l}\text { Non-APAP } \\
\text { initial } \\
\text { survivors } \\
(N=200)\end{array}$ & $\begin{array}{l}\text { LT recipient } \\
(N=262)\end{array}$ & $P$-value \\
\hline \multicolumn{6}{|l|}{ Demographics } \\
\hline Age (years) & 768 & $37.5 \pm 12.5$ & $42.6 \pm 15.2$ & $38.9 \pm 13.7$ & $<0.001$ \\
\hline$\%$ Female & 768 & $235(76.8)$ & $122(61.0)$ & $167(63.7)$ & $<0.001$ \\
\hline \multicolumn{6}{|l|}{ Race } \\
\hline \% Caucasian & 768 & $266(86.9)$ & $138(69.0)$ & $183(69.8)$ & \multirow[t]{3}{*}{$<0.001$} \\
\hline$\%$ Black & & $23(7.5)$ & $41(20.5)$ & $46(17.6)$ & \\
\hline$\%$ Other & & $17(5.6)$ & $21(10.5)$ & $33(12.6)$ & \\
\hline Ethnicity (\% Not Hispanic/Latino) & 768 & $293(95.8)$ & $182(91.0)$ & $231(88.2)$ & 0.004 \\
\hline \multicolumn{6}{|l|}{ Marital status } \\
\hline$\%$ Married & 495 & $78(39.6)$ & $58(52.3)$ & $108(57.8)$ & \multirow[t]{3}{*}{0.01} \\
\hline$\%$ Never married & & $79(40.1)$ & $37(33.3)$ & $54(28.9)$ & \\
\hline$\%$ Other & & $40(20.3)$ & $16(14.4)$ & $25(13.4)$ & \\
\hline \multicolumn{6}{|l|}{ Employment } \\
\hline$\%$ Employed & 768 & $67(21.9)$ & $50(25.0)$ & $114(43.5)$ & \multirow[t]{3}{*}{$<0.001$} \\
\hline$\%$ Other & & $44(14.4)$ & $33(16.5)$ & $43(16.4)$ & \\
\hline$\%$ Unknown & & $195(63.7)$ & $117(58.5)$ & $105(40.1)$ & \\
\hline Years of education & 474 & $12.5 \pm 2.4$ & $12.9 \pm 2.6$ & $13.5 \pm 2.6$ & 0.001 \\
\hline \multicolumn{6}{|l|}{ Medical history } \\
\hline \multicolumn{6}{|l|}{ Aetiology } \\
\hline$\%$ APAP & 764 & $306(100.0)$ & $0(0.0)$ & $33(12.8)$ & \multirow[t]{8}{*}{$<0.001 *$} \\
\hline$\%$ Autoimmune hepatitis & & $0(0.0)$ & $21(10.5)$ & $37(14.3)$ & \\
\hline \% DILI & & $0(0.0)$ & $48(24.0)$ & $55(21.3)$ & \\
\hline$\%$ Hepatitis A & & $0(0.0)$ & $13(6.5)$ & $7(2.7)$ & \\
\hline \% Hepatitis B & & $0(0.0)$ & $22(11.0)$ & $32(12.4)$ & \\
\hline \% Shock/lschaemia & & $0(0.0)$ & $38(19.0)$ & $1(0.4)$ & \\
\hline$\%$ Indeterminate & & $0(0.0)$ & $33(16.5)$ & $68(26.4)$ & \\
\hline$\%$ Other & & $0(0.0)$ & $25(12.5)$ & $25(9.7)$ & \\
\hline Symptoms to ALF (days) & 739 & $3.0 \pm 2.0$ & $10.0 \pm 14.0$ & $17.0 \pm 23.2$ & $<0.001$ \\
\hline Jaundice to ALF (days) & 635 & $1.0 \pm 2.0$ & $5.0 \pm 8.5$ & $11.5 \pm 17.0$ & $<0.001$ \\
\hline Weight (kg) & 728 & $72.5 \pm 19.8$ & $80.6 \pm 21.3$ & $80.2 \pm 20.7$ & $<0.001$ \\
\hline$\%$ Psychiatric disease & 768 & $167(54.6)$ & $54(27.0)$ & $34(13.0)$ & $<0.001$ \\
\hline$\%$ Substance abuse & 768 & $140(45.8)$ & $29(14.5)$ & $21(8.0)$ & $<0.001$ \\
\hline$\%$ History of IDU & 756 & $41(13.6)$ & $16(8.1)$ & $4(1.6)$ & $<0.001$ \\
\hline \% Hypertension & 768 & $32(10.5)$ & $53(26.5)$ & $34(13.0)$ & $<0.001$ \\
\hline \% Endocrine/Diabetes & 768 & $31(10.1)$ & $40(20.0)$ & $34(13.0)$ & 0.006 \\
\hline$\%$ Heart disease & 768 & $14(4.6)$ & $25(12.5)$ & $11(4.2)$ & $<0.001$ \\
\hline \multicolumn{6}{|l|}{ Presenting labs } \\
\hline AST (IU/L) & 765 & $2805 \pm 6300$ & $1048 \pm 2070$ & $626 \pm 1431$ & $<0.001$ \\
\hline $\operatorname{ALT}(I \cup / L)$ & 761 & $3272 \pm 4226$ & $1454 \pm 2358$ & $766 \pm 1762$ & $<0.001$ \\
\hline INR & 759 & $2.5 \pm 2.0$ & $2.1 \pm 1.2$ & $3.1 \pm 2.3$ & $<0.001$ \\
\hline Bilirubin (mg/dl) & 764 & $4.1 \pm 3.6$ & $9.9 \pm 14.6$ & $22.1 \pm 16.3$ & $<0.001$ \\
\hline MELD & 754 & $27.8 \pm 8.9$ & $29.3 \pm 7.1$ & $34.8 \pm 7.4$ & $<0.001$ \\
\hline Phosphate (IU/L) & 655 & $2.3 \pm 1.8$ & $3.3 \pm 2.1$ & $3.3 \pm 2.1$ & $<0.001$ \\
\hline Creatinine (mg/dl) & 767 & $1.4 \pm 2.1$ & $1.4 \pm 1.9$ & $1.1 \pm 1.6$ & 0.055 \\
\hline Lactate (mmol/L) & 396 & $3.2 \pm 3.6$ & $3.5 \pm 3.3$ & $4.9 \pm 6.7$ & $<0.001$ \\
\hline$\%$ Urine tox screen positive & 768 & $180(58.8)$ & $48(24.0)$ & $48(18.3)$ & $<0.001$ \\
\hline \multicolumn{6}{|l|}{ Clinical complications at admission } \\
\hline$\%$ Pressors & 748 & $32(10.6)$ & $29(14.9)$ & $37(14.7)$ & 0.259 \\
\hline$\%$ Intubated & 766 & $130(42.5)$ & $72(36.2)$ & $95(36.4)$ & 0.228 \\
\hline$\%$ Grade $3 / 4 \mathrm{HE}$ & 766 & $134(43.8)$ & $70(35.2)$ & $98(37.5)$ & 0.115 \\
\hline \% Mannitol & 759 & $29(9.6)$ & $13(6.5)$ & $37(14.4)$ & 0.02 \\
\hline \% ICP monitor & 705 & $21(7.3)$ & $11(6.0)$ & $31(13.3)$ & 0.015 \\
\hline \% Dialysis/CVVH & 753 & $63(20.8)$ & $28(14.2)$ & $51(20.2)$ & 0.150 \\
\hline \multicolumn{6}{|l|}{ Peak labs } \\
\hline ALT (IU/L) & 768 & $3677 \pm 4669$ & $1479 \pm 2391$ & $939 \pm 2071$ & $<0.001$ \\
\hline INR & 763 & $2.8 \pm 2.3$ & $2.4 \pm 1.1$ & $3.8 \pm 2.9$ & $<0.001$ \\
\hline
\end{tabular}


Table 1 (continued)

\begin{tabular}{|c|c|c|c|c|c|}
\hline & $N$ & $\begin{array}{l}\text { APAP } \\
\text { initial } \\
\text { survivors } \\
(N=306)\end{array}$ & $\begin{array}{l}\text { Non-APAP } \\
\text { initial } \\
\text { survivors } \\
(N=200)\end{array}$ & $\begin{array}{l}\text { LT recipient } \\
(N=262)\end{array}$ & $P$-value \\
\hline Bilirubin (mg/dl) & 768 & $7.0 \pm 8.2$ & $14.2 \pm 16.4$ & $24.8 \pm 14.0$ & $<0.001$ \\
\hline MELD & 763 & $29.6 \pm 9.2$ & $31.0 \pm 7.2$ & $37.1 \pm 7.4$ & $<0.001$ \\
\hline Creatinine (mg/dl) & 768 & $1.9 \pm 3.9$ & $1.7 \pm 2.8$ & $1.4 \pm 2.1$ & 0.041 \\
\hline Lactate $(\mathrm{mmol} / \mathrm{L})$ & 449 & $3.3 \pm 3.9$ & $3.7 \pm 3.6$ & $4.9 \pm 6.6$ & $<0.001$ \\
\hline \multicolumn{6}{|c|}{ Clinical complications during ALF hospitalization } \\
\hline$\%$ Pressors & 768 & $57(18.6)$ & $38(19.0)$ & $71(27.1)$ & 0.029 \\
\hline$\%$ Intubated & 768 & $160(52.3)$ & $85(42.5)$ & $159(60.7)$ & $<0.001$ \\
\hline$\%$ Grade $3 / 4 \mathrm{HE}$ & 766 & $162(52.9)$ & $89(44.7)$ & $149(57.1)$ & 0.03 \\
\hline \% Mannitol & 768 & $51(16.7)$ & $19(9.5)$ & $58(22.1)$ & 0.001 \\
\hline$\%$ ICP monitor & 768 & $36(11.8)$ & $13(6.5)$ & $60(22.9)$ & $<0.001$ \\
\hline \% Dialysis/CVVH & 768 & $99(32.4)$ & $52(26.0)$ & $86(32.8)$ & 0.222 \\
\hline
\end{tabular}

*Compares the proportion of non-APAP aetiologies between the non-APAP SS and LT recipients.

DILI, drug-induced liver injury; IDU, intravenous drug use.

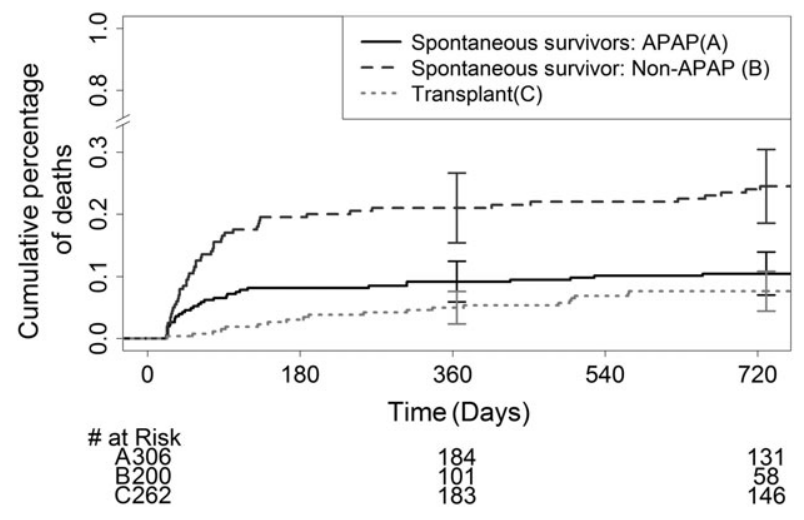

Fig. 2. Actuarial 2-year mortality among the 773 initial 3-week survivors. The liver transplant recipients $(C)$ had the highest 2-year survival $(92.4 \%)$ compared to the acetaminophen overdose (A) spontaneous survivors $(89.5 \%)$ and the non-acetaminophen (B) spontaneous survivors $(75.0 \%)(P<0.0001)$. those who died varied, but there was no specific nonAPAP aetiology which was over-represented in those who died vs. survived (Table S2a, $P=0.35$ ). Of the 49 patients who died with non-APAP ALF, only five $(10.2 \%)$ were listed for liver transplant, while 32 of the 151 survivors $(21.1 \%)$ were initially listed for LT during their hospitalization.

\section{Liver transplant recipients}

In the multivariate model for LT recipients, age, days from jaundice to ALF onset and higher coma grade remained as consistent predictors of death with older patient age indicating higher risk of death (10 years OR: 1.53, 95\% CI: 1.06, 2.21). There did not appear to be an over-representation of any particular aetiology of ALF in those who died vs. survived (Table S2b, $P=0.07$ ). However, $12 \%$ of the APAP LT recipients died as well as $11 \%$ of the autoimmune patients and $5 \%$ of the druginduced liver injury (DILI) patients. Although the proportion of patients with advanced encephalopathy (Grade $3 / 4$ ) at presentation was significant on univariate analysis, this did not persist in multivariate analysis. The

Table 2. Causes of death in ALF patients

\begin{tabular}{|c|c|c|c|}
\hline & $\begin{array}{l}\text { APAP initial } \\
\text { survivors }(N=32)\end{array}$ & $\begin{array}{l}\text { Non-APAP initial } \\
\text { survivors }(N=49)\end{array}$ & LT recipients $(N=20)$ \\
\hline$\%$ Liver related & 9.4 & 18.4 & 25.0 \\
\hline$\%$ Infection/sepsis & 12.5 & 12.2 & 10.0 \\
\hline$\%$ Cardiac & 0.0 & 8.2 & 10.0 \\
\hline$\%$ Neurological & 6.3 & 4.1 & 10.0 \\
\hline$\%$ Multisystem organ failure & 28.1 & 8.2 & 25.0 \\
\hline$\%$ Other & 12.5 & 6.1 & 15.0 \\
\hline \% Unknown & 40.6 & 53.1 & 30.0 \\
\hline Days to death & $57.0(84.0)$ & $57.0(95.0)$ & $222.5(380.5)$ \\
\hline Median (IQR)* & & & \\
\hline
\end{tabular}

$\star P<0.001$ Patients can have multiple causes of death. 
Table 3. Univariate and multivariate models of 2-year survival in the three ALF patient groups

\begin{tabular}{|c|c|c|c|c|}
\hline & Univariate odds ratio $(95 \% \mathrm{Cl})^{*}$ & $P$-value & Multivariate odds ratio $(95 \% \mathrm{Cl})^{*}$ & $P$-value \\
\hline \multicolumn{3}{|l|}{ APAP initial spontaneous survivors } & \multicolumn{2}{|l|}{ C-Statistic: $0.647(0.539,0.755)$} \\
\hline Age (per 10 years) & $1.34(1.02,1.76)$ & 0.038 & $1.27(0.95,1.71)$ & 0.110 \\
\hline ALT (per 50 IU/L) & $0.994(0.987,1.001)$ & 0.071 & - & - \\
\hline Phosphate (mg/dl) & $1.28(1.07,1.54)$ & 0.007 & $1.26(1.05,1.51)$ & 0.015 \\
\hline Arterial pH & $0.04(0.001,1.125)$ & 0.059 & - & - \\
\hline Initial HE (\%3/4) & $2.02(0.96,4.26)$ & 0.064 & - & - \\
\hline Pressor use & $2.73(1.07,6.95)$ & 0.035 & - & - \\
\hline Dialysis/CVVH use & $1.87(0.84,4.18)$ & 0.128 & - & - \\
\hline \multicolumn{3}{|c|}{ Non-APAP initial spontaneous survivors } & \multicolumn{2}{|l|}{ C-Statistic: $0.687(0.596,0.778)$} \\
\hline Age (per 10 years) & $1.44(1.15,1.80)$ & 0.001 & $1.54(1.17,2.02)$ & 0.002 \\
\hline Ethnicity (\% Hisp/Lat) & $0.36(0.08,1.62)$ & 0.183 & - & - \\
\hline Education ( $\%<12$ years) & $2.16(0.76,6.10)$ & 0.148 & - & - \\
\hline Days from jaundice to ALF & $1.03(1.01,1.06)$ & 0.015 & $1.03(1.00,1.06)$ & 0.028 \\
\hline ALT (per 50 IU/L) & $0.987(0.978,0.997)$ & 0.013 & - & - \\
\hline INR & $0.83(0.64,1.06)$ & 0.138 & - & - \\
\hline Pressor use & $1.77(0.76,4.14)$ & 0.185 & - & - \\
\hline \multicolumn{3}{|l|}{ Liver transplant recipients } & \multicolumn{2}{|l|}{ C-Statistic: $0.741(0.613,0.869)$} \\
\hline Age (10 years) & $1.43(1.02,2.01)$ & 0.036 & $1.53(1.06,2.21)$ & 0.024 \\
\hline Days from jaundice to ALF & $0.97(0.93,1.01)$ & 0.113 & $0.97(0.93,1.01)$ & 0.162 \\
\hline Days from symptoms to ALF & $0.98(0.95,1.01)$ & 0.18 & - & - \\
\hline Initial HE (\%3/4) & $2.70(1.06,6.87)$ & 0.037 & $2.57(0.94,7.05)$ & 0.066 \\
\hline INR & $0.78(0.58,1.05)$ & 0.104 & - & - \\
\hline MELD & $0.96(0.90,1.02)$ & 0.173 & - & - \\
\hline Intubation use & $2.28(0.91,5.73)$ & 0.078 & - & - \\
\hline Dialysis/CVVH use & $2.31(0.87,6.14)$ & 0.092 & - & - \\
\hline
\end{tabular}

*Odds are modelled as the probability of death.

median age of the LT recipients who died was 45.5 compared to 38 years in those who survived. The median days to jaundice of the LT recipients who died was 6 compared to 12 in those who survived. The proportion of LT recipients who died that had grade $3 / 4 \mathrm{HE}$ was $60 \%$ compared to $36 \%$ in those who survived.

\section{Long-term clinical and functional outcomes}

Assessment of long-term functional outcomes are reported in Table 4 from the 353 patients who provided follow-up data in person or via telephone interview including 123 APAP initial survivors, 85 non-APAP initial survivors and $145 \mathrm{LT}$ recipients. The rate of employment, marriage and having private insurance was lowest in the APAP patients compared to others. In the subgroup of patients with an available Karnofsky score, the median score among the APAP initial survivors was lower than the other two groups but this difference was not statistically significant.

\section{Medical and psychiatric comorbidities during long-term follow-up}

The frequency of diabetes, hypertension and hyperlipidaemia in the three groups were similar at the last available long-term follow-up study visit (Table 4). However, ongoing psychiatric illness was significantly more common in the APAP SS patients compared to the other groups. Furthermore, problematic alcohol consumption was significantly more commonly reported in the APAP group compared to the others and intentional drug overdose/suicide was also significantly more common in the APAP SS patients.

\section{Liver disease and laboratory status}

The median values of serum AST, ALT, alk phos and bilirubin were normal at the last available follow-up visit in the three subgroups of patients. However, the median serum total bilirubin levels were significantly lower in the LT recipients compared to the other patient groups while the median serum creatinine levels were significantly higher in the LT recipients (Table 4).

\section{Discussion}

ALF is a rare but potentially devastating illness that frequently afflicts previously healthy and young individuals. The ALFSG and other groups have previously reported that 3-week transplant-free survival is significantly higher in subjects with APAP overdose, hepatitis A, and pregnancy-related ALF ( $\sim 60$ to $70 \%$ ) compared to subjects with DILI, Wilson's disease and indeterminate ALF ( 20 to $40 \%)(1-4)$. In addition, the ALFSG demonstrated that patient age, initial and peak grade of hepatic encephalopathy, and selected laboratory variables are associated with a greater likelihood of death during the initial ALF hospitalization (1,2). Currently, 
Table 4. Functional status at last follow-up visit among ALF long-term survivors

\begin{tabular}{|c|c|c|c|c|c|}
\hline & $N^{*}$ & $\begin{array}{l}\text { APAP } \\
\text { initial } \\
\text { survivors } \\
(N=123)\end{array}$ & $\begin{array}{l}\text { Non-APAP } \\
\text { initial } \\
\text { survivors } \\
(N=85)\end{array}$ & $\begin{array}{l}\mathrm{LT} \text { recipients } \\
(N=145)\end{array}$ & $P$-value \\
\hline \multicolumn{6}{|l|}{ Demographics } \\
\hline Time of form collection (days) & 276 & $704 \pm 435$ & $732 \pm 424$ & $746 \pm 400$ & 0.191 \\
\hline Age (years) & 353 & $37.5 \pm 12.8$ & $42.2 \pm 13.9$ & $39.2 \pm 13.0$ & 0.044 \\
\hline$\%$ Female & 353 & $95(77.2)$ & $53(62.4)$ & $95(65.5)$ & 0.040 \\
\hline \multicolumn{6}{|l|}{ Marital status } \\
\hline$\%$ Married & 247 & $24(30.8)$ & $33(54.1)$ & $56(51.9)$ & \multirow{3}{*}{0.025} \\
\hline$\%$ Never married & 247 & $34(43.6)$ & $19(31.1)$ & $30(27.8)$ & \\
\hline$\%$ Other & 247 & $20(25.6)$ & $9(14.8)$ & $22(20.4)$ & \\
\hline \multicolumn{6}{|l|}{ Employment } \\
\hline \% Employed & 276 & $27(29.0)$ & $30(45.5)$ & $53(45.3)$ & \multirow[t]{3}{*}{$<0.001$} \\
\hline$\%$ Other & 276 & $35(37.6)$ & $26(39.4)$ & $52(44.4)$ & \\
\hline$\%$ Unknown & 276 & $31(33.3)$ & $10(15.2)$ & $12(10.3)$ & \\
\hline Years of education & 197 & $11.8 \pm 2.3$ & $12.7 \pm 2.3$ & $12.6 \pm 3.2$ & 0.181 \\
\hline$\%$ Uninsured & 276 & $14(15.1)$ & $5(7.6)$ & $2(1.7)$ & 0.001 \\
\hline$\%$ Private insurance & 276 & $36(38.7)$ & $45(68.2)$ & $73(62.4)$ & $<0.001$ \\
\hline \% Medicare & 276 & $16(17.2)$ & $9(13.6)$ & $17(14.5)$ & 0.796 \\
\hline$\%$ Medicaid & 276 & $12(12.9)$ & $5(7.6)$ & $18(15.4)$ & 0.312 \\
\hline Karnofsky score & 197 & $86 \pm 16$ & $90 \pm 12$ & $90 \pm 11$ & 0.148 \\
\hline \multicolumn{6}{|l|}{ Substance abuse at last follow-up visit } \\
\hline $\begin{array}{l}\text { Time of form collection (days) } \\
\% \text { Suicide attempt }\end{array}$ & 344 & $762 \pm 408$ & $734 \pm 443$ & $746 \pm 346$ & 0.38 \\
\hline No & 325 & $80(72.7)$ & $59(76.6)$ & $124(89.9)$ & \multirow[t]{3}{*}{0.004} \\
\hline Yes & 325 & $7(6.4)$ & $2(2.6)$ & $3(2.2)$ & \\
\hline Unknown & 325 & $23(20.9)$ & $16(20.8)$ & $11(8.0)$ & \\
\hline \multicolumn{6}{|l|}{$\%$ Psychiatric disease } \\
\hline No & 326 & $40(36.0)$ & $48(62.3)$ & $96(69.6)$ & \multirow[t]{3}{*}{$<0.001$} \\
\hline Yes & 326 & $45(40.5)$ & $14(18.2)$ & $31(22.5)$ & \\
\hline Unknown & 326 & $26(23.4)$ & $15(19.5)$ & $11(8.0)$ & \\
\hline \multicolumn{6}{|l|}{$\%$ Alcohol abuse } \\
\hline No & 325 & $71(64.0)$ & $55(72.4)$ & $126(91.3)$ & \multirow[t]{3}{*}{$<0.001$} \\
\hline Yes & 325 & $14(12.6)$ & $4(5.3)$ & $1(0.7)$ & \\
\hline Unknown & 325 & $26(23.4)$ & $17(22.4)$ & $11(8.0)$ & \\
\hline \multicolumn{6}{|c|}{ Medical complications at last follow-up visit } \\
\hline Time of form collection (days) & 353 & $763 \pm 375$ & $738 \pm 444$ & $746 \pm 370$ & 0.601 \\
\hline$\%$ Neurological complications & 353 & $33(39.3)$ & $16(26.7)$ & $42(32.6)$ & 0.276 \\
\hline$\%$ Diabetes mellitus & 270 & $8(9.8)$ & $8(13.3)$ & $22(17.2)$ & 0.314 \\
\hline \% Hypertension & 256 & $15(18.8)$ & $13(22.0)$ & $27(23.1)$ & 0.763 \\
\hline \% Hyperlipidaemia & 267 & $9(11.4)$ & $7(11.7)$ & $9(7.0)$ & 0.454 \\
\hline \multicolumn{6}{|l|}{ Most recent labs } \\
\hline Time of last Lab (days) & 320 & $224 \pm 672$ & $264 \pm 695$ & $709 \pm 449$ & $<0.001$ \\
\hline Median AST (IU/L) & 286 & $27 \pm 46$ & $30 \pm 34$ & $31 \pm 27$ & 0.669 \\
\hline$\%$ AST > ULN & 286 & $41(45.6)$ & $26(42.6)$ & $69(51.1)$ & 0.491 \\
\hline Median ALT (IU/L) & 287 & $39 \pm 99$ & $28 \pm 44$ & $31 \pm 33$ & 0.613 \\
\hline$\% A L T>U L N$ & 287 & $47(52.2)$ & $26(41.9)$ & $58(43.0)$ & 0.316 \\
\hline Median alk phos (IU/L) & 226 & $82 \pm 33$ & $82 \pm 69$ & $89 \pm 78$ & 0.336 \\
\hline$\%$ Alk phos $>$ ULN & 226 & $13(20.3)$ & $14(31.1)$ & $33(28.2)$ & 0.383 \\
\hline Median T. bilirubin (mg/dl) & 284 & $0.7 \pm 1.3$ & $0.8 \pm 2.0$ & $0.6 \pm 0.4$ & 0.015 \\
\hline$\%$ Bilirubin > ULN & 284 & $30(33.7)$ & $24(40.0)$ & $22(16.3)$ & $<0.001$ \\
\hline Median creatinine (mg/dl) & 295 & $0.8 \pm 0.6$ & $0.9 \pm 0.5$ & $1.1 \pm 0.5$ & $<0.001$ \\
\hline$\%$ Creatinine $>$ ULN & 295 & $20(21.3)$ & $9(14.3)$ & $39(28.3)$ & 0.082 \\
\hline Median haemoglobin (g/dl) & 291 & $12.1 \pm 2.8$ & $12.9 \pm 3.2$ & $12.7 \pm 2.1$ & 0.033 \\
\hline$\%$ Haemoglobin < LLN & 291 & $73(76.8)$ & $38(64.4)$ & $96(70.1)$ & 0.237 \\
\hline Median platelet count $\left(\times 10^{3} / \mathrm{ml}\right)$ & 293 & $248 \pm 140$ & $210 \pm 137$ & $212 \pm 89$ & 0.011 \\
\hline$\%$ Platelet count < LLN & 293 & $13(13.8)$ & $14(23.0)$ & $22(15.9)$ & 0.313 \\
\hline
\end{tabular}

*Patient population defined by collection of outcome data at in person or via phone with chart review.

The following ULN values were used: AST ULN 30 IU/L, ALT ULN 35 IU/L, alk phos ULN 130 IU/L, bilirubin ULN 1.2 mg/dl, creatinine ULN 1.3 mg/dl. The following LLN were used: Haemoglobin LLN $13.5 \mathrm{~g} / \mathrm{dl}$, platelet count LLN 150. 
many clinicians use these parameters and other laboratory and clinical features at the bedside to help guide decisions regarding the need to list and proceed with LT $(3,4)$. However, the long-term clinical outcomes in ALF patients who survive their initial illness are not well described. Understanding long-term outcomes in initial survivors with ALF has proven difficult because of the low incidence of the disease in the general population with only 2000-3000 annual cases in the USA.

In this study, we were able to determine the 2-year clinical outcomes in 773 ALF patients who had survived their initial illness. The patients included in this analysis are generally representative of the entire ALFSG cohort, although fewer APAP patients were included compared to the overall cohort (Table S1). Liver transplantation yielded the highest 2-year survival at $92.4 \%$ compared to the other two groups of initial spontaneous survivors. These results are somewhat surprising as subjects who require an emergency LT tend to be sicker at presentation compared to subjects that spontaneously recover and would be expected to continue to have a substantial risk of death during prolonged follow-up. The greater initial severity of illness at presentation among the LT recipients is supported by the higher initial and peak serum bilirubin, INR, lactate and MELD scores in these patients compared to the other two groups (Table 1). The 20 deaths in the LT recipients occurred throughout the 2 years of follow-up, while most of the deaths in the initial spontaneous survivors tended to occur within 6 months of enrolment into the ALFSG study, suggesting that ongoing hepatic damage may lead to fatal outcomes after the initial illness. In attempting to understand the reasons for the absolute differences in mortality and time to death, we first looked at the causes of death in the three patient groups. There was no apparent differences in the stated causes of death but there were moderate number of 'unknown' causes in each subgroup (Table 2). A recent study of hospitalized adult patients with APAP overdose in Canada demonstrated that many of the patients died during 5 years of follow-up from a variety of preventable causes including trauma, substance abuse and other high-risk behaviours (13). In addition, the survival of the APAP overdose patients was significantly lower than the expected rate in age-matched population controls. Therefore, the observation that roughly $10 \%$ of our APAP initial survivors died during 2 years of follow-up is not entirely surprising. Our data also suggest that the increased frequency of medical and psychiatric comorbidities in these highrisk patients may require additional treatments and interventions to improve long-term outcomes.

To further explore the observed differences in mortality, models of 2-year patient mortality were constructed using baseline variables in all three patient subgroups (Table 3). Of note, a higher patient age at admission was significantly associated with impaired survival in both the LT and non-APAP initial survivor groups. These data are consistent with results of prior studies of LT recipients which have also demonstrated that increased patient age is a strong negative predictor of survival in ALF patients (14-16). Other studies have also demonstrated that liver regeneration is impaired in older livers $(14,17)$. Therefore, our data are consistent with prior studies demonstrating the importance of subject age in surviving severe acute liver injury. In each of the subgroups, specific baseline features were identified as being associated with impaired survival on multivariate analysis (Table 3). Among the APAP patients, a higher phosphate level was associated with poorer survival which is consistent with prior studies demonstrating that hyperphosphataemia is associated with poorer short-term outcomes $(18,19)$. This association may be because of impaired utilization of phosphate in liver regeneration or simply as a marker of patients with poorer renal function. Unfortunately, serial serum phosphate levels during the ALF hospitalization or during long-term follow-up were not available for testing. In addition, a lower arterial $\mathrm{pH}$ and higher arterial lactate levels are important predictors of short-term outcomes in the King's college criteria and arterial $\mathrm{pH}$ and INR were significant but only on univariate analysis in our cohort $(3,4)$. However, we did not detect a poorer long-term survival in APAP patients with more advanced encephalopathy at presentation. Nonetheless, other studies have demonstrated that severe cerebral oedema is predictive of poor neurological outcomes and survival in ALF patients $(20,21)$.

Among the non-APAP initial survivors, age, serum ALT levels, and a longer duration of symptoms were all identified on univariate analyses as associated with a lower likelihood of long-term survival $(3,21-24)$. The ALFSG has previously shown that Hispanic patients are more likely to have a lower transplant-free survival rate compared to non-Hispanic patients in part because of their greater likelihood of having DILI (25). However in multivariate analysis, only patient age and a longer duration of symptoms to ALF onset were significant and independent predictors of death. A poorer outcome in patients with subacute ALF that present with more slowly but inevitably progressive liver injury than those with hyperacute ALF has been previously reported (26, 27). Furthermore, the majority of these non-APAP patients were never listed for LT and nearly $20 \%$ had ALF because of ischaemic hepatitis which is associated with severe underlying cardiopulmonary disease that frequently precludes LT (10).

Among the LT recipients, patient age and more advanced encephalopathy at presentation were associated with poorer 2-year survival on univariate analyses. These data are consistent with prior studies that demonstrate the critical importance of the initial and peak encephalopathy grade in predicting outcome in ALF (3, $28,29)$. However, in multivariate analysis only age and the days from jaundice to ALF onset remained independent predictors of outcomes. Although other studies have demonstrated that LT recipients with APAP overdose are at increased risk of graft failure compared to 
other ALF patients, we were not able to identify a relationship between the aetiology of ALF and death during prolonged follow-up possibly because of the limited number of deaths in this population.

A careful review of the functional outcomes in the three patient groups demonstrated some important and interesting differences (Table 4). For example, LT recipients were significantly more likely to be employed compared to the other two groups both at presentation and during follow-up. These observations may in part be because of the lower socioeconomic status of patients who present with APAP overdose compared to the other two groups. Although nearly $40 \%$ of the APAP overdose patients were non-intentional 'therapeutic misadventures' who received toxic doses of multiple APAP products there was a significantly higher rate of concomitant substance abuse and psychiatric disease requiring treatment in the APAP SS group during follow-up compared to the other two groups which was independent of intentionality (29). In addition, $6.1 \%$ of the APAP initial survivors reported another episode of drug overdose/suicide which is also consistent with prior studies of repeated high-risk behaviours in this patient population (13).

Recent studies have demonstrated that LT recipients with ALF have a lower rate of employment compared to cirrhotic LT recipients despite their younger age (30). In addition, ALF patients experience higher rates of depression, anxiety, and post-traumatic stress disorder after liver transplantation compared to cirrhotic LT recipients (31). Whether this is because of the sudden onset of a severe life-threatening illness in generally young and previously well ALF patients compared to chronically ill cirrhotic LT recipients is not known but plausible. The Karnofsky scores which are indicative of global health status tended to be higher in the LT recipients compared to the other two groups although the difference did not reach statistical significance. In a recent analysis of health related quality of life measures in a subgroup of ALFSG long-term survivors, we showed that the LT recipients had significantly higher SF-36 and CDC-14 scores during follow-up compared to the APAP and non-APAP initial survivors (32). These cumulative data suggest that both APAP and non-APAP initial survivors appear to have poorer general physical and mental health compared to the LT recipients during longterm follow-up in addition to a lower overall survival. As such, a significant subset of ALF spontaneous survivors may benefit from targeted interventions including counselling, psychiatric care, and physical therapy to achieve improved long-term health outcomes.

The median serum AST, ALT, alk phos, and bilirubin levels were normal in all of the patients in each of the three patient subgroups during their most recent follow-up visit (Table 4). The significantly lower total bilirubin levels in the LT recipients is somewhat surprising and suggests that the APAP and non-APAP initial survivors may have poorer bilirubin clearance. However, our study was not designed to assess liver disease status via histological sampling or radiological imaging to further explore this observation. We surmise that the higher mean serum creatinine levels in the LT recipients likely relates to the use of nephrotoxic calcineurin inhibitors to prevent rejection. However, only $28 \%$ of the LT recipients had an elevated serum creatinine level so the degree of renal insufficiency appears to be mild. In addition, the incidence of diabetes and hypertension was generally low and similar in the three patient groups.

Limitations of this study include the incomplete follow-up of all 1172 eligible ALF survivors at 3 weeks (Fig. 1). One of the reasons for incomplete follow-up is that over $80 \%$ of the ALF patients are transferred to an ALFSG site for tertiary liver care. For patients that survive their index hospitalization with no evidence of residual liver injury, continued follow-up at a referral centre that may be several hours from their home, can be challenging. This may in part explain the larger proportion of APAP initial survivors who did not return for follow-up (Table S1). In addition, continued participation in a non-interventional registry study would be expected to have a lower rate of follow-up than a clinical trial. Lastly, $45 \%$ of the ALF patients that died during prolonged follow-up did not have an identifiable cause of death (Table 2). This rate of incomplete data is similar to those seen in other population and cohort-based studies of long-term outcomes of patients $(33,34)$. The incomplete reporting of the causes of death in our study may again relate to the absence of long-term medical follow-up in the liver centre because of logistical issues as well as our inability to routinely obtain Death Certificates or causes of death from the Social Security Death Index. In addition, the declining use of autopsy in patients that die in a hospital setting and the difficulty to accurately ascertain the cause of death in individuals without an autopsy may have played a role $(33,35,36)$. Despite these limitations, our study represents the largest cohort of adult ALF patients that were prospectively followed beyond 21 days and up to 2 years. In addition, the severity of illness and other clinical features of the enrolled patients were generally similar to the excluded patients. Another limitation of this study was that we did not account for donor, operative or immunosuppression variables when attempting to explore outcomes in the LT recipients. This was because of the fact that our study was not intended to standardize the treatment or monitoring of ALF patients after LT. Furthermore, the limited number of deaths in the LT recipients may have precluded adequate power to identify other predictors of long-term outcomes.

In conclusion, our study results demonstrate that the 2-year clinical outcomes in early survivors with ALF are generally very good. Although nearly $40 \%$ of initial adult ALF patients die during their index hospitalization, the mortality rate among initial 3-week survivors is substantially lower during more prolonged follow-up. Contrary to our initial hypothesis, the nonAPAP initial survivors have a significantly lower 2-year 
survival compared to the LT recipients and APAP initial survivors. Most of the deaths in the initial spontaneous survivors occurred within the first 6 months of observation because of a multitude of causes suggesting that early careful follow-up after an ALF episode is warranted. Among the long-term survivors, the APAP initial survivors had the lowest rates of employment which may be because of their lower baseline socioeconomic status and more frequent medical and psychiatric comorbidities compared to the other patient groups. Laboratory markers of liver injury demonstrated normal or near normal parameters in most patients during follow-up. Therefore, despite the need for intubation, dialysis and pressors in many ALF patients during their acute illness, most will recover with intact liver function during follow-up. Although mortality is highest in the non-APAP initial survivors, the overall favourable 2-year survival can help guide future studies of additional interventions to improve clinical outcomes in these patients.

\section{Acknowledgements}

Members and institutions participating in the Acute Liver Failure Study Group 1998-2010 are as follows: W.M. Lee, M.D. (Principal Investigator), George A. Ostapowicz, M.D., Frank V. Schiødt, M.D., Julie Polson, M.D., University of Texas Southwestern, Dallas, TX; Anne M. Larson, M.D., Iris Liou, M.D., University of Washington, Seattle, WA; Timothy Davern, M.D., University of California, San Francisco, CA (current address: California Pacific Medical Center, San Francisco, CA), Oren Fix, M.D., University of California, San Francisco; Michael Schilsky, M.D., Mount Sinai School of Medicine, New York, NY (current address: Yale University, New Haven, CT); Timothy McCashland, M.D., University of Nebraska, Omaha, NE; J. Eileen Hay, M.B.B.S., Mayo Clinic, Rochester, MN; Natalie Murray, M.D., Baylor University Medical Center, Dallas, TX; A. Obaid S. Shaikh, M.D., University of Pittsburgh, Pittsburgh, PA; Andres Blei, M.D., Northwestern University, Chicago, IL (deceased), Daniel Ganger, M.D., Northwestern University, Chicago, IL; Atif Zaman, M.D., University of Oregon, Portland, OR; Steven H.B. Han, M.D., University of California, Los Angeles, CA; Robert Fontana, M.D., University of Michigan, Ann Arbor, MI; Brendan McGuire, M.D., University of Alabama, Birmingham, AL; Raymond T. Chung, M.D., Massachusetts General Hospital, Boston, MA; Alastair Smith, M.B., Ch.B., Duke University Medical Center, Durham, NC; Robert Brown, M.D., Cornell/ Columbia University, New York, NY; Jeffrey Crippin, M.D., Washington University, St Louis, MO; Edwin Harrison, Mayo Clinic, Scottsdale, AZ; Adrian Reuben, M.B.B.S., Medical University of South Carolina, Charleston, SC; Santiago Munoz, M.D., Albert Einstein Medical Center, Philadelphia, PA; Rajender Reddy, M.D., University of Pennsylvania, Philadelphia, PA; R.
Todd Stravitz, M.D., Virginia Commonwealth University, Richmond, VA; Lorenzo Rossaro, M.D., University of California Davis, Sacramento, CA; Raj Satyanarayana, M.D., Mayo Clinic, Jacksonville, FL; and Tarek Hassanein, M.D., University of California, San Diego, CA. The University of Texas Southwestern Administrative Group included Grace Samuel, Ezmina Lalani, Carla Pezzia, and Corron Sanders, Ph.D., Nahid Attar, the Statistics and Data Management Group included Joan S. Reisch, Ph.D., Linda S. Hynan, Ph.D., Janet P. Smith, Joe W. Webster and Mechelle Murray, and the Medical University of South Carolina Data Coordination Unit included Valerie Durkalski, Ph.D., Wenle Zhao, Ph.D., Catherine Dillon, and Tomoko Goddard.

Financial support: We gratefully acknowledge the support provided by the members of The Acute Liver Failure Study Group. This study was funded by the National Institute of Diabetes, Digestive and Kidney Diseases (DK U-01-58369). Additional funding provided by the Tips Fund of Northwestern Medical Foundation and the Jeanne Roberts and Rollin and Mary Ella King Funds of the Southwestern Medical Foundation. In addition, Dr. Rangnekar is supported by the T32 DK62708-01, NIDDK Training Grant in Gastrointestinal Epidemiology, and a Clinical and Translational Science Award from the Michigan Institute for Clinical and Health Research.

Conflict of interest: Drs. Rangnekar, Sanders, Hynan, Reuben, Liou, Han, Schilsky, Reuben, Stravitz, Fix, Davern, and Durkalski and Ms Ellerbe have no financial conflicts of interest. Dr. Fontana has served as a consultant to Bristol-Meyers Squibb, Vertex Pharmaceuticals, Tibotec, Merck, GlaxoSmithkline and Medtronic in the past year. Reddy Consultant: Vertex, Janssen, Merck, Genentech-Roche, BMS, Idenix, Gilead, Novartis Investigator: Vertex, Janssen, BMS, Abbott, Ikaria; McGuireGrant support (Bayer, Cumberland, Ikaria, Vertex), consultant (Hepahope); Ganger -Consultant (speaker) to Vertex, Gilead, and Otsuka; Chung-Grant support (Gilead, Mass Biologics, Merck), consultant (Enanta, Idenix); Lee-Consultant (Lilly, Novartis, Cumberland) research support (BMS, Gilead, Merck, Roche, Boeringher- Ingleheim, Andays, Vertex).

\section{References}

1. Stravitz RT, Kramer AH, Davern T, et al. Intensive care of patients with acute liver failure: recommendations of the US Acute Liver Failure Study Group. Crit Care Med 2007; 35: 2498-2508.

2. Ostapowicz G, Fontana RJ, Schiodt FV, et al. Results of a prospective study of acute liver failure at 17 tertiary care centers in the United States. Ann Intern Med 2002; 137: 947-954.

3. O'Grady JG, Alexander GJ, Hayllar KM, Williams R. Early indicators of prognosis in fulminant hepatic failure. Gastroenterology 1989; 97: 439.

4. Bernal W, Donaldson N, Wyncoll D, Wendon J. Blood lactate as an early predictor of outcome in paracetamol- 
induced acute liver failure: a cohort study. Lancet 2002; 359: 558-563.

5. Freeman RB, Steffick DE, Guidinger MK, et al. Liver and intestine transplantation in the United States, 1997-2006. Am J Transplant 2008; 8: 958-976.

6. Mohamed R, Hubscher SG, Mirza DF, Gunson BK, Mutimer DJ. Postransplantation chronic hepatitis in fulminant hepatic failure. Hepatology 1997; 25: 1003-1007.

7. Devlin J, Williams R. Transplantation for fulminant hepatic failure. Transplantation 1996; 62: 151-155.

8. Jackson EW, Zacks S, Zinn S, et al. Delayed neuropsychological dysfunction after liver transplantation for acute liver failure: a matched case-controlled study. Liver Transpl 2002; 10: 932-936.

9. Lewis $\mathrm{MB}$, Howdie PD. Cognitive dysfunction and HRQOL in long-term liver transplant survivors. Liver Transpl 2003; 9: 1145-1148.

10. Taylor R, Tujios S, Jinjuvadia K, et al. Short and longterm outcomes in patients with acute liver failure due to ischemic hepatitis. Dig Dis Sci 2012; 57: 777-784.

11. Stravitz RT, Lefkowitch JH, Fontana RJ, et al. Autoimmune acute liver failure: proposed clinical and histological criteria. Hepatology 2010; 53: 517-526.

12. Hanley JA, McNeil B. The meaning and use of the area under an ROC curve. Radiology 1982; 143: 29-36.

13. Myers RP, Shaheen AM, Li B, Dean S, Quan H. Impact of liver disease, alcohol abuse, and unintentional ingestions on the outcomes of acetaminophen overdose. Clin Gastro Hep 2008; 6: 918-926.

14. Germani G, Theocharidou E, Adam R, et al. Liver transplantation for acute liver failure in Europe: outcomes over 20 years from the ELTR database. J Hepatol 2012; 57: 288296.

15. Bernal W, Cross TJ, Auzinger G, et al. Outcome after waitlisting for emergency liver transplantation in acute liver failure: a single center experience. J Hepatol 2009; 50: 306-313.

16. Barshes NR, Lee TC, Balkrishnan R, et al. Risk stratification of adult patients undergoing liver transplantation for fulminant hepatic failure. Transplantation 2006; 81: 195-201.

17. Yamashiki N, Sugawara Y, Tamura S, et al. Outcomes after living donor liver transplantation for acute liver failure in Japan: results of a Nationwide Survey. Liver Transpl 2012; 18: 1069-1077.

18. Schmidt LE, Dalhoff K. Serum phosphate is an early predictor of outcome in severe acetaminophen-induced hepatotoxicity. Hepatology 2002; 36: 659-663.

19. Macquillan GC, Seyam MS, Nightingale P, Neuberger JM, Murphy N. Blood lactate but not serum phosphate levels can predict patient outcome in fulminant hepatic failure. Liver Transpl 2005; 11: 1073-1079.

20. Chan G, Taqui A, Marotta P, et al. Long-term outcomes of emergency liver transplantation for acute liver failure. Liver Transpl 2009; 15: 1696-1702.

21. Tan WF, Steadman RH, Farmer DG, et al. Pretransplant neurological presentation and severe posttransplant brain injury in patients with acute liver failure. Transplantation 2012; 94: 768-774.

22. Bjornsson E, Olsson R. Outcome and prognostic markers in severe drug-induced liver disease. Hepatology 2005; 42: 481-489.

23. Gimson AE, O'Grady J, Ede RJ, Portman B, Williams R. Late-onset hepatic failure: clinical, serological and histological features. Hepatology 1986; 6: 288.
24. Rautou PE, Moucari R, Cazals-Hatem D, et al. Levels and initial course of serum alanine aminotransferase can predict outcome of patients with Budd-Chiari syndrome. Clin Gastroenterol Heptol 2009; 7: 1230-5.

25. Forde KA, Reddy KR, Troxel AB, Sanders CM, Lee WM. Racial and ethnic differences in presentation, etiology, and outcomes of acute liver failure in the US. Clin Gastro Hep 2009; 7: 1121-1126.

26. Dhiman RK, Makharia GK, Jain S, Chawla Y. Ascites and spontaneous bacterial peritonitis in fulminant hepatic failure. Am J Gastroenterol 2000; 95: 233.

27. Vaquero J, Polson J, Chung C, et al. Infection and the progression of hepatic encephalopathy in acute liver failure. Gastroenterology 2003; 125: 755-764.

28. McDiarmid SV, Goodrich NP, Harper AM, Merion RM. Liver transplantation for status 1: the consequences of good intentions. Liver Transpl 2007; 13: 699-707.

29. Larson AM, Polson J, Fontana RJ, et al. Acetaminopheninduced acute liver failure: results of a United States multicenter, prospective study. Hepatology 2005; 42: 1364-72.

30. Aberg F, Hockerstedt K, Roine RP, Sintonen H, Isoniemi $H$. Influence of liver-disease etiology on long-term quality of life and employment after liver transplantation. Clin Transplant 2012; 26: 729-735.

31. Guimaro MS, Lacerda SS, Aguilar MR, et al. Post-traumatic stress disorders, mood disorders, and quality of life in transplant recipients with acute liver failure. Transplant Proc 2011; 43: 187-188.

32. Rangnekar AS, Ellerbe C, Durkalski V, et al. Quality of life is significantly impaired in long-term survivors of acute liver failure and particularly in acetaminophen overdose patients. Liver Transpl 2013; 19: 991-1000.

33. Helweg-Larsen K. The Danish register of causes of death. Scan J Pub Health 2011; 39(Suppl 7): 26-29.

34. Potterat JJ, Brewer DD, Muth SQ, et al. Mortality in a long-term open cohort of prostitute women. Am J Epidemiol 2004; 159: 778-785.

35. Reidel D, Mwangi E, Fantry L, et al. High cancer-related mortality in an urban, predominantly African-American, HIV-infected population. AIDS 2013; 27: 1109-1107.

36. Asnaes S. Uncertainity of determining mode and cause of death without autopsy: an autopsy study of medically unattended non-medicolegal deaths. For Sci Int 1980; 15: 191-196.

\section{Supporting information}

Additional Supporting Information may be found in the online version of this article:

Table S1. Comparison of baseline characteristics among those included in the long-term follow-up ALF study and those excluded.

Table S2a. Proportion of deaths after 3 weeks within an etiology for Non-APAP initial survivors.

Table S2b. Proportion of deaths after 3 weeks within an etiology for LT recipient patients.

Table S3. Functional status at last follow-up visit among ALF long-term survivors (Information is form dependent). 